\title{
REVIEW
}

\section{Ageing and vascular ageing}

\author{
B Jani, C Rajkumar
}

Postgrad Med J 2006;82:357-362. doi: 10.1136/pgmj.2005.036053

There is an age related decline in various physiological processes. Vascular ageing is associated with changes in the mechanical and the structural properties of the vascular wall, which leads to the loss of arterial elasticity and reduced arterial compliance. Arterial compliance can be measured by different parameters like pulse wave velocity, augmentation index, and systemic arterial compliance. There is evidence that arterial compliance is reduced in disease states such as hypertension, diabetes, and end stage renal failure. Changes in arterial compliance can be present before the clinical manifestation of cardiovascular disease. Pharmacological and non-pharmacological measures have been shown to improve arterial compliance. Arterial compliance may constitute an early cardiovascular risk marker and may be useful in assessing the effects of drugs on the cardiovascular system. Pharmacogenetics and genetics of arterial compliance in the future will improve our knowledge and understanding about vascular ageing.

See end of article for authors' affiliations

Correspondence to: Dr B Jani, Section of Care of the Elderly, Imperial College, Hammersmith Hospital, Du Cane Road, London W12 ONN, UK; binitajani@hotmail.com

Submitted 13 April 2005 Accepted 16 July 2005
$\mathrm{T}$ here is an age related decline in various physiological body processes. Chronological age may be different from biological age. Some institutions offer biological testing where they measure lung capacity, memory, reaction time, and hearing and visual reaction speed to assess biological age. Certain biological variables like greying of hair and skin elasticity correlate in a linear fashion with chronological age. ${ }^{1}$ Estimates of arterial function (vascular ageing) have consistently been found to be related closely to the chronological age as Thomas Sydenham said, "A man is as old as his arteries". ${ }^{2}$

Vascular ageing contributes to the age dependent rise in hypertension and atherosclerotic disease. Ageing in itself confers a greater risk for the disease than the conventional risk factors such as lipid levels, smoking, diabetes, and sedentary lifestyle.

The most noticeable characteristic of vascular ageing is the change in the mechanical and structural properties of the vascular wall. Arteries have intrinsic and functional properties that are subject to lifelong stress and hormonal changes. These properties are changed in the presence of modifiable cardiovascular (CV) risk factors such as hypertension, obesity, smoking, and lifestyle, and non-modifiable risk factors such as age, genetics, and family history.

The arterial system is composed of a branching network of elastic conduits (aorta) and high resistance vessels (small arterioles and capillaries), which constitute a hydraulic filter converting the intermittent output from the heart into steady capillary flow. The arterial pulse wave form is derived from the complex interaction of the left ventricular stroke volume, the physiological properties of the arterial tree (arterial compliance), and the viscous properties of blood. Blood pressure (BP) has two components-the pulsatile component (pulse pressure (PP)) determined by left ventricular ejection (LVE) and arterial compliance and the steady state component (mean arterial pressure (MAP)) maintained by the small peripheral arteries. There is accumulating evidence suggesting abnormalities in the arterial pulsatile characteristics occur early in the disease process associated with increased CV risk, for example, hypertension and diabetes, and can be favourably modified by therapeutic interventions.

Arterial compliance is defined as a change in area, diameter, or volume of an artery or arterial bed for a given change in pressure. ${ }^{3}$ Arterial compliance can be measured non-invasively using indirect measures such as pulse wave velocity (PWV), augmentation index (AI), pulse contour analysis, and echo vascular wall tracking which will be discussed later. Reduced arterial compliance has been shown to be associated with increased CV mortality and morbidity. From the Framingham risk estimates, conventional risk factors do not explain all the risk and vascular compliance may be one of the missing CV risk factor. ${ }^{4}$

\section{ARTERIAL SYSTEM}

The arterial system consists of the larger elastic arteries and the smaller muscular peripheral arteries. The arterial wall consists of three concentric layers-the tunica adventitia, tunica media, and tunica intima. There is a single mono layer of endothelial cells between the blood and the vessel wall. Large arteries are rich in elastin and collagen and small muscular arteries are rich in vascular smooth muscle. Elastin fibres play an important part in determining the mechanical strength of the vessels at lower pressures and

Abbreviations: PWV, pulse wave velocity; Al, augmentation index; SAC, systemic arterial compliance; CV, cardiovascular; LVE, left ventricular ejection; MAP, mean arterial pressure; PP, pulse pressure; CIMI, carotid intimal thickness; IMT, intimal thickness; ACEl, angiotensin converting enzyme inhibitor; SBP, systolic blood pressure; DBP, diastolic blood pressure; ESRF, end stage renal failure; CRP, C reactive protein; HRT, hormone replacement therapy; BP, blood pressure

This article is part of a series on ageing edited by Professor Chris Bulpitt. 
collagen fibres bear most of the strength at the higher pressures. $^{5}$

Large elastic arteries such as the aorta buffer the flow and the pressure variation generated by the intermittent left ventricular contraction and convert the pulsatile variation into the steady flow to the peripheries. This maintains continuous oxygenation to the tissues and minimises cardiac work. Elastic recoil of the central arteries in diastole is important for coronary perfusion. Loss of this elasticity impairs coronary flow and may contribute to coronary artery disease. In young people BP is determined largely by peripheral vascular resistance and in older people it is also determined by large vessel stiffness.

The pulse wave is formed by the overlapping of the two waves - the forward wave and the reflected wave. The forward wave travels from the heart to the periphery (depends on LVE and arterial stiffness) and the reflected wave travels from the periphery to the heart. It is possible to evaluate the timing and magnitude of the reflected wave.

The effects of ageing on the arterial tree are heterogeneous and the mechanical properties of the blood vessels vary depending on the vascular territory studied-that is, difference between elastic and muscular arteries. ${ }^{6}$

\section{STRUCTURAL CHANGES THAT OCCUR IN THE ARTERIAL TREE WITH AGEING}

In the larger elastic arteries there is increase in collagen content, covalent cross linking of the collagen, elastin fracture, and calcification and reduction in the elastin content with ageing. There are also changes in endothelial function, wall thickness media to lumen ratio, and arterial stiffness with ageing.

Endothelium changes arterial structure and function by producing vaso-active substances like nitric oxide (potent vasodilator) and endothelin (potent vaso-constrictor and procoagulant). With age, endothelin production increases and nitric oxide production decreases. This favours a procoagulant state and promotes vascular smooth muscle growth. In disease states such as hypertension and diabetes this sequence is exaggerated causing an increased risk of $\mathrm{CV}$ events. $^{7}$

Postmortem studies show that aortic wall thickening with ageing consists of an increase in intimal thickness even in populations with a low incidence of atherosclerosis. ${ }^{8}$ Carotid intimal thickness (CIMT) increases threefold between ages 20-90 years. Excess intimal thickness (IMT) at a given age predicts silent coronary artery disease and this will be accelerated in presence of known CV risk factors. It has been shown that pharmacological interventions with statins and angiotension converting enzyme inhibitors (ACEI) and life style changes can slow the progression of intima medial thickness.

\section{FUNCTIONAL CHANGES WITH AGEING: CHANGES IN ARTERIAL COMPLIANCE}

Compliance is a functional property of the artery and it reduces with age as mentioned earlier. Compliance is defined as the unit change in volume induced by a unit change in pressure.

The age related increase in systolic blood pressure (SBP) is greater compared with the increase in diastolic blood pressure (DBP). Reduced compliance causes earlier return of the reflected wave and this wave arrives in systole rather than diastole and causes a disproportionate rise in systolic pressure and increases PP. This increases cardiac work load and reduces coronary flow in diastole.

Isolated systolic hypertension (ISH) and PP are late surrogate measures of reduced compliance and are associated with increased risk of CV events.

\section{MEASURES OF ARTERIAL COMPLIANCE}

There are invasive (direct intra-arterial measurements) and non-invasive methods to measure compliance. Non-invasive methods have been shown to correlate well with invasive methods. Some of the commonly used non-invasive methods include PWV, AI, and systemic arterial compliance (SAC). Other methods used are echo tracking, digital pulse contour analysis, and QKD (Q-wave to diastolic Korotkoff sound) interval.

\section{PWV}

The velocity of the pulse wave between two points in the vasculature, PWV is measured in metres/second is closely related to the arterial segment measured. Two pulse or flow wave forms are recorded at known distance apart (D) to accurately measure the time delay between the recorded waves by applanation tonometry. The transit time $(\Delta \mathrm{T})$ is calculated from the foot of the first wave recorded at the proximal site and the foot of the second wave recorded at the distal end. $\mathrm{PWV}=\mathrm{D} / \Delta \mathrm{T}$. PWV depends on the viscoelastic properties of the wall and the blood density as shown by the Moens-Korteweg equation and the Bramwell-Hill equation. ${ }^{1}$ PWV is sensitive to heart rate and BP and has to be adjusted for these variables. PWV is an easy and reproducible method that can be used in clinical practice. Increased PWV reflects three potential risk factors - that is, increased SBP, increased PP, and changed wall properties.

Central (aortic) PWV has been shown to predict CV morbidity and mortality in high risk patients with hypertension and end stage renal failure (ESRF). Central aortic compliance is reduced in presence of coronary artery disease and left ventricular hypertrophy. ${ }^{10}$

An increase of $1 \mathrm{~m} / \mathrm{s}$ in aortic PWV has been shown to equate to $39 \%$ increase in risk of CV events. ${ }^{11}{ }^{12}$ Muscular arteries do not behave in the same way as elastic arteries. ${ }^{6}$ Changes in peripheral PWV do not correlate well with disease states. Diabetic patients and patients with ESRF have been shown to have a higher central PWV compared with age matched healthy people. Higher aortic PWV has been shown to predict $\mathrm{CV}$ mortality in the elderly population. ${ }^{13}$

\section{Al}

It is defined as $\Delta \mathrm{p} / \mathrm{pp}$ (where $\Delta \mathrm{p}$ is the difference between the late systolic peak and the mid-systolic peak and $\mathrm{pp}$ is the amplitude of the pulse pressure wave), and permits the estimation of the effect of the reflected wave on the amplitude of the pulse pressure wave. AI can be obtained by application of a transfer function to the pressure pulse recorded at the radial or carotid artery by applanation tonometry. We can use the Sphygmocor Atcor medical device to obtain AI using the above method. In older people with stiffer arteries the reflected wave arrives in systole and causes systolic hypertension. AI depends on PWV and BP and is inversely proportional to heart rate and height. It is a measure of cardiac workload.

\section{SAC}

It is a surrogate measure of central arterial compliance. This is achieved by simultaneous recording of the ascending aortic blood flow with a Doppler velocimetry transducer applied on the suprasternal notch and the PPV of the right carotid artery by applanation tonometry. Arterial compliance is calculated using the formula derived by Liu et al. ${ }^{14}$ The diameter of the aortic valve is measured using $2 \mathrm{D}$ echocardiography.

\section{Echo tracking}

Echo tracking studies local arterial wall properties using measurement of the separation of the anterior and posterior walls of an artery plotted against time during the cardiac systole. This is time consuming and requires a high degree of 
technical expertise. Other methods will not be discussed in detail here.

\section{ARTERIAL COMPLIANCE IN DISEASE STATES}

Diabetes is associated with a two to three times increase in $\mathrm{CV}$ events. It is also associated with obesity, dyslipidaemia, hypertension, and renal failure. In type 2 diabetes there is evidence of formation of advanced glycation end products (AGEs), which lead to structural changes in the vessel wall. ${ }^{15}{ }^{16}$ Hyperglycaemia, dyslipidaemia, hypertension, and hyperinsulinaemia all promote endothelial dysfunction and contribute to arterial stiffness.

PWV is increased in conditions such as ESRF, diabetes, ${ }^{17}$ familial hypercholesterolaemia, ${ }^{18}$ and Marfan's syndrome. There is increased mortality in patients with ESRF and a higher aortic PWV that is independent of the other risk factors. Lower extremity arterial compliance and central compliance measures have been associated with the presence of significant coronary artery stenoses in patients undergoing cardiac catheterisation. ${ }^{19} 20$

In patients with essential hypertension increase in carotid femoral PWV of $3.5 \mathrm{~m} / \mathrm{s}$ is associated with 34\% increased relative risk of primary coronary events as shown in the first longitudinal prospective study. ${ }^{21}$ Laurent et al have shown an increase in aortic PWV of $5 \mathrm{~m} / \mathrm{s}$ is associated with a $34 \%$ increase in all cause mortality and a $51 \%$ increase in $\mathrm{CV}$ mortality. ${ }^{22}$ After age 70 there is $19 \%$ increase in CV risk per $1 \mathrm{~m} / \mathrm{s}$ increase in aortic PWV. ${ }^{23}$

\section{SEX DIFFERENCES IN ARTERIAL FUNCTION}

There are differences in body weight, height, body fat distribution, heart rate, stroke volume, and arterial compliance between the two sexes. In the very elderly, age related large artery stiffness and pulsatility is more pronounced in women. This may be attributable to smaller body size and arterial dimensions independent of menopause. ${ }^{24}$ Pre-pubertal women have stiffer arteries and higher PP than their pre-pubertal male counterparts. Post-puberty arteries are more distensible in women than men and the risk of $\mathrm{CV}$ disease is much lower in pre-menopausal women. ${ }^{25} 26$ This may represent intrinsic differences in arterial properties between men and women. SAC and AI correlate with height in men and women. SAC is positively and AI is negatively correlated with body mass index.

Distribution of body fat may have an effect on arterial compliance and glucose metabolism. Larger trunk fat mass is associated with high peripheral arterial stiffness and larger lower leg fat mass and leg lean mass is associated with lower peripheral arterial stiffness. Trunk or leg fat mass is not associated with central arterial compliance. ${ }^{27}$ Higher fat levels in the leg is associated with lower blood glucose levels and higher trunk fat mass is associated with higher blood glucose levels after adjustment for age, trunk lean mass, leg lean mass, and leg fat mass. ${ }^{28}$

\section{GENETICS AND VASCULAR AGEING}

There is evidence that the relation between genotypes and arterial stiffness may manifest itself in later life.

Two distinct groups of genes have been implicated in arterial stiffness-those that are associated with cell signalling (vascular cell adhesion molecules) and those that are associated with the regulation of vascular structure (cytoskeletal-cell membrane and the extra cellular matrix). The MMP-3 genotype may be an important determinant of arterial stiffness, with heterozygotes having the optimal balance of matrix accumulation and deposition. ${ }^{29}$ The T-allele has been associated with greater MMP-9mRNA and protein levels and T-allele carriers have greater large artery stiffness because of excessive degradation of the arterial elastic matrix. $^{30}$ MMP-3 genotype determined is by $5 \mathrm{~A} / 6 \mathrm{~A}$ polymorphism and 5A/5A homozygous is linked with increased stiffness and increased SBP. ${ }^{31}$ The ATl receptor genotype may also influence arterial ageing in hypertensive people. ${ }^{32}$

There are also protective genetic factors that result in a delayed onset of vascular disease even in the presence of smoking and high cholesterol (longevity genes). ${ }^{33}$ AfroCaribbeans have a high prevalence of hypertension and diabetes and diabetic patients have been shown to have reduced peripheral compliance. This may account for higher rates of cerebrovascular events in Afro-Caribbeans although they have lower rates of coronary heart disease. ${ }^{34} 35$

\section{RELATION BETWEEN ATHEROSCLEROSIS AND ARTERIAL STIFFNESS}

Atherosclerosis is a patchy disease and increased PWV, a measure of arterial stiffness, represents a composite measure of the segment under evaluation. This probably identifies a more extensive calcific disease distributed through the arterial tree. The Rotterdam study, which was a population based study, showed increased arterial stiffness was associated with atherosclerosis at various sites. ${ }^{36}$

Atherosclerosis is an inflammatory process related to endothelial dysfunction and excess deposition of oxidised lipids. There is active process of injury and healing within the endothelium and smooth muscle and it is the balance of this process that dictates the progression of atheroma. In most people this process progresses through lifetime, so by age 50 most will have coronary involvement. Rate of progression is dictated by risk factors such as smoking, dyslipidaemia, diabetes, and hypertension. Atherosclerosis leads to thick stiff arterial walls and calcification and plaque formation and this would change the mechanical properties of the arteries. ${ }^{37}$ Arterial plaque formation can be measured by ultrasound techniques and CIMT has been used as a marker of atherosclerosis in some studies. Aortic and brachial PWV and PP relate to level of inflammation measured by high sensitivity $\mathrm{C}$ reactive protein (CRP) levels in healthy people suggesting that inflammation may be involved in arterial stiffening. There may be a potential role of anti-inflammatory drugs in reducing premature vascular ageing. ${ }^{38}$ Statins have been shown to reduce inflammation and improve vascular compliance. Increased arterial stiffness and atherosclerosis may coexist or may be two independent markers of subclinical vascular damage in young patients. ${ }^{39}$

\section{HOW CAN WE MODIFY COMPLIANCE?}

There is convincing evidence that treating known vascular risk factors such as diabetes, hypertension, obesity, dyslipidaemia, and smoking reduces risk of CV events by up to $20 \%-$ $30 \%$. Pharmacological and non-pharmacological measures improve brachial BP, HbAlc, and lipid levels and also improve arterial compliance independent of the above parameters.

There is evidence that arterial compliance can be modified by drugs, diet, and lifestyle changes.

\section{Drugs}

Antihypertensive drugs improve mean arterial BP, endothelial function, vessel tone, and reduce structural remodelling. In most of the published studies ACEIs, diuretics, and calcium channel blockers improve PWV, AI, and SAC over and above $\mathrm{BP}$ reduction in long term studies. ACEIs improve age dependent IMT and smooth muscle hypertrophy. Nonselective $\beta$ blockers do not improve arterial compliance as much as other drugs. ${ }^{40-42}$

Nitrates improve compliance acutely and after long term use. The new nitric oxide donor sinitrodil improves arterial compliance in healthy young men in doses that do not affect 
resistance vessels. ${ }^{43}$ In congestive cardiac failure nitrates and ACEIs are effective in improving the SAC. ACEI efficacy may be influenced by genetic polymorphism. Patients carrying the C-allele of the Al166C polymorphism of the angiotensin 2 ATl receptor were shown to have improved arterial stiffness after perindopril compared with the calcium antagonist nitrendipine, and the reverse was seen with the homozygote $\mathrm{M}$ allele. ${ }^{44}$

Statins undoubtedly reduce CV mortality in high risk group people. Use of statins in secondary prevention is well established and there is growing evidence for use of statins in primary prevention in high risk people. Statins improve lipid levels, improve endothelial function, reduce inflammation in the atherosclerotic plaques, stabilise the plaques, and have been shown to reduce progression of IMT in coronary artery disease. Statins improve large artery stiffness in patients with familial hypercholesterolaemia and to a lesser extent in ISH. $^{44}$ In a large cohort of elderly hypertensive patients plasma total cholesterol was not associated with large artery stiffness.$^{45}$ Statins reduce CRP levels. Raised CRP levels have been associated with poor prognosis postmyocardial infarction, and any adverse prognosis in vascular disease. $^{46}$

\section{Exercise}

Aerobically trained athletes have a better SAC then matched sedentary controls. In some studies exercise in the elderly improves PP, PWV, AI, baroreceptor function, and endothelial function. ${ }^{47}$ Weight lifters, on the other hand, have lower arterial compliance. ${ }^{48}$ Weight loss in healthy obese men induced a significant reduction in mean BP that was associated with an improvement in large artery compliance. Aerobic training was not shown to modify large artery compliance in older patients with $\mathrm{ISH}^{49}$

\section{Hormone replacement therapy (HRT)}

There are sex differences in arterial compliance as discussed earlier. Oestrogen therapy has been shown to reduce LDL cholesterol, increase HDL cholesterol, and may also inhibit lipoprotein oxidation and proliferation of smooth muscle cells in the arterial wall. There is variable evidence for improvement in arterial function after use of HRT. HRT has been shown in some trials to improve SAC and IMT after adjustments for lipid profiles while others have shown no improvement in arterial stiffness and endothelial dysfunction. HRT has not been shown to improve BP in 24 hour ambulatory BP monitoring. ${ }^{50-53}$ Hypoandrogenaemia in men and hyperandrogenaemia in women has been shown to be associated with increased risk of coronary events..$^{54}$

\section{Dietary modifications}

Arterial compliance in obese subjects improves with dietary plant n-3 fatty acids from flax seed oil despite an increase in LDL-oxidisabilty. ${ }^{56}$

Fish oils have been shown to increase HDL cholesterol, reduce triacylglycerol rich lipoprotein, reduce post-prandial lipaemia, and improve endothelial function and arterial compliance in type 2 diabetic patients and non-diabetic patients. ${ }^{57-59}$

Soya-dietary isoflavins (phytoestrogens) have been shown to reduce the progression of atherosclerosis and improve arterial stiffness in older women. ${ }^{6061}$ Phytoestrogens are plant derivatives structurally similar to oestrogen that are ligands for the oestrogen receptors ER $\alpha$ and ER $\beta$. Soya bean and red clover are rich sources of isoflavins-a subgroup of phytoestrogens that include diadzeim, formonotin, genistin, and their precursors. Epidemiological studies have suggested a reduction in $\mathrm{CV}$ risk in population consuming a high isoflavin diet (Shanghai women's health study). In studies using the formonotin isoflavin showed improvement in SAC, central PWV, and reduced peripheral resistance but no improvement in the endothelial function. This isoflavin is not found in soybeans. ${ }^{63}$

Low salt diet improves arterial function and lowers BP in older patients with systolic hypertension. ${ }^{63}$

Vitamin $\mathrm{C}$ has not been shown to improve arterial compliance. ${ }^{64}$

\section{Smoking}

Few studies have investigated the effects of long term smoking on arterial wall properties. The results are contradictory depending on methods used. Smoking is however a known risk factor for atherosclerosis and may contribute to arterial stiffness. ${ }^{65}$

\section{WHY DO WE NEED TO MEASURE COMPLIANCE?}

There is evidence that subclinical vascular disease is present and can be assessed before the overt clinical manifestation. Brachial BP has been traditionally used to stratify CV risk. Changes in the mechanical properties in the presence of unchanged brachial BP have been shown by an increase in SAC with aerobic fitness and HRT in post-menopausal women. Drugs like ACEIs improve AI and PWV and nitrates improve AI independent of the BP reduction. ${ }^{40-42}$

Arterial stiffness may play a potential aetiological part in $\mathrm{CV}$ disease, may constitute an early risk marker, and may be useful in assessing the effects of drugs on the CV system. Measures to reduce arterial compliance may modify progression before a CV event occurs. Methods to measure compliance are simple, reliable, and can be used in the clinical setting.

\section{VASCULAR AGEING AND FUTURE DEVELOPMENTS}

With more research and genetics unfolding we will have more insight into how to improve arterial compliance and how it correlates to the clinical CV risk. This may provide insight into concept of successful compared with unsuccessful vascular ageing. One target approach would be to interfere with collagen metabolism but collagen turnover is a slow process, and studies looking at this would be difficult to design. Pharmacogenetics would be an interesting area in future to predict a person's drug responsiveness.

Aldosterone is an important mediator of fibrotic changes at the level of the heart and recent data suggest that similar effects may occur in the arterial walls of rats and humans. ${ }^{66}$ Nitric oxide donors seem a potential group of therapeutic agents.

Advanced glycation end products (AGE) link proteins have been shown to increase arterial stiffness in diabetes and ageing. Drugs interfering with formation of AGE link products, such as amino guanidine and ALT-711, have been shown in animal models to reverse arterial stiffening with changes in BP. ALT-711 has been shown to improve compliance in elderly people. These drugs may prove useful in diabetic and hypertensive patients. ${ }^{67}$

\section{CONCLUSIONS}

- Age is an important non-modifiable CV risk factor. ${ }^{68}$ Improving arterial compliance reduces risk of $\mathrm{CV}$ events-primary and secondary prevention.

- Non-invasive methods to assess arterial biomechanics and functional properties are available. Arterial compliance may be one of the missing risk factor in global risk CV risk stratification.

- Arterial compliance can be modified by therapeutic interventions and it would be possible to identify people with early vascular damage who are predisposed to future vascular events. 
- We need more longitudinal population based studies and randomised controlled trials to show that progressive disease and pharmacological and non-pharmacological measures are accompanied by an adverse or favourable outcome.

\section{Authors' affiliations}

B Jani, Section of Care of the Elderly, Imperial College, London, UK C Rajkumar, Faculty of Medicine, Brighton and Sussex Medical School, Brighton, UK

Funding: none.

Conflicts of interest: none.

\section{REFERENCES}

1 Bulpitt CJ, Shipley MJ, Broughton PMG, et al. The assessment of biological age. A report from the department of environment study. Ageing Clin Exp Res 1994;6:181-91.

2 Anon. Thomas Sydenham (1624-1689). Quoted in Bulletin of the New York. Acad Med 1928:4:993.

3 McVeigh GE, Hamilton PK, Morgan DR. Evaluation of mechanical arterial properties: clinical, experimental and therapeutics aspects. Clin Sci 2002;102:51-67.

4 Hodes RJ, Lakatta EG, Meneil CT. Another modifiable risk factor for cardiovascular disease? Some evidence points to arterial stiffness. J Am Geriatr Soc 1995;43:581-2.

5 Bank AJ, Wong H, Holte JE, et al. Contribution of collagen, elastin and smooth muscle in vivo human arterial wall stress and elastic modulus. Circulation 1994: 12:3263-70

6 McVeigh GE, Bratteli CW, Morgan DJ, et al. Age related abnormalities in arterial compliance identified by pressure pulse contour analysis: ageing and arterial compliance. Hypertension 1999;33:1392-8.

7 McVeigh GE, Alen PB, Morgan DR, et al. Nitric oxide modulation of blood vessel tone identified by arterial wave form analysis. Clin Sci 2001;100:387-93.

8 Virmani R, Avolio AP, Mergner WJ, et al. Effect of ageing on aortic morphology in populations with high and low prevalence of hypertension and atherosclerosis. Comparison between occidental and Chinese communities. Am J Pathol 1991;139:1119-29.

9 Cameron JD, Jennings GL, Dart AM. The relationship between arterial compliance, age, blood pressure and serum lipid levels. J Hypertens 1995:13:1718-23.

10 Bulpitt CJ, Cameron JD, Rajkumar C, et al. The effect of age on vascular compliance in man: Which are the appropriate measures? J Hum Hypertens 1999;13:753-8.

11 Blacher J, Guerin AP, Pannier B, et al. Impact of aortic stiffness on survival in end stage renal failure. Circulation 1999:99:2434-9.

12 Safar ME, Blacher J, Pannier B, et al. Central Pulse pressure and mortality in end stage renal failure. Hypertension 2002;39:735-8.

13 Meauma S, Benetos A, Henly OF, et al. Aortic PWV predicts cardiovascular mortality in subjects over 70 years of age. Atheroscler Thromb Vasc Biol $2001 \cdot 21 \cdot 2046-50$

14 Liv Z, Brin KP, Yin FC. Estimation of total arterial compliance: an improved method and evaluation of current methods. Am J Physiol 1986;251:588-600.

15 Cameron JD, Pinto E, Bulpitt CJ, et al. The ageing of elastic and muscular arteries: a comparison of diabetic and non-diabetic subjects. Diabetes Care 2003;26:2127-32.

16 Pinto E, Mensah R, Meeran K, et al. Peripheral Arterial Compliance differs between races-comparison among Asian, Afro-Caribbeans, and white Caucasians with type 2 diabetes. Diabetes Care 2005;28:496.

17 Lehmann ED, Gosling RG, Sonksen PH. Arterial wall compliance in diabetes. Diabet Med 1992;9:114-19.

18 Lehmann ED, Watts GF, Fatemi-Langroudi B, et al. Aortic compliance in young patients with heterozygous familial hypercholesterolemia. Clin Sci 1992:83:717-21.

19 Syeda B, Gottsauner-Wolf M, Denk S, et al. Arterial compliance: a diagnostic marker for atherosclerotic plaque burden? Am J Hypertens 2003;16:356-62.

20 Herrington DM, Kesler K, Reiber JC, et al. Arterial compliance adds to conventional risk factors for presence of angiographic coronary artery disease. Am Heart J 2003;146:662-7.

21 Boutouyrie P, Tropeano Al, Asmar R, et al. Aortic stiffness is an independent predictor of primary coronary events in hypertensive patients: a longitudinal study. Hypertension 2002;39:10-15.

22 Laurent S, Boutouyrie P, Asmar R, et al. Aortic stiffness is an independent predictor of all cause and cardiovascular mortality in hypertensive patients. Hypertension 2001;37:1236-41.

23 Meaume S, Benetos A, Henry OF, et al. Aortic pulse wave velocity predicts cardiovascular mortality in subjects $>70$ years of age. Arterioscler Thromb Vasc Biol 2001:21:2046-50.

24 Smulyen H, Asmar RG, Ridnicki A, et al. Comparative effects of ageing in men and women on the properties of the arterial tree. J Am Coll Cardiol 2001;37:1374-81.

25 Ahimastos AA, Formosa M, Dart AM, et al. Gender differences in large artery stiffness pre-and post puberty. J Clin Endocrinol Metab 2003;88:5375-80.
26 Bulpitt CJ, Rajkumar C, Cameron JD. Vascular Compliance as a measure of biological age. J Am Geriatr Soc 1999;47:657-63.

27 Snijder MB, Henry RM, Visser M, et al. Regional body composition as a determinant of arterial stiffness in the elderly: the HOORN study. J Hypertens 2004;229:2339-47.

28 Snijder MB, Dekker JM, Visser M, et al. Trunk fat and leg fat have independent and opposite association with fasting and post load glucose levels. The HOORN study. Diabetes Care 2004;27:372-7.

29 Medley TL, Kingwell BA, Gatzka CD, et al. MMP3 genotype contributes to age related aortic stiffening through modulation of gene and protein expression. Circ Res 2003;92:1254-61

30 Medley TL, Cole TJ, Dart AM, et al. Matrix metalloproteinase-9 genotype influences large artery stiffness through effects on aortic gene and protein expression. Arterioscler Thromb Vasc Biol 2004;24:1479-84.

31 Kingwell BA, Medley TL, Waddell TK, et al. Large artery stiffness: structural and genetic aspects. Clin Exp Pharmacol Physiol 2001;28:1040-3.

32 Lajemi M, Labat C, Gautier S, et al. Angiotensin 2 type 1 receptor-153 A/G and $1166 \mathrm{~A} / \mathrm{C}$ gene polymorphisms and increase in aortic stiffness with age in hypertensive subjects. J Hypertens 2001;19:7-13.

33 Slagboom PE, Heijmans BT, Beekman M, et al. Genetics of human ageing: the search for genes contributing to human longevity and disease of the old. Ann N Y Acad Sci 2000;908:50-63

34 Chaturvedi N, Bulpitt CJ, Leggetter S, et al. Ethnic differences in vascular stiffness and relations to hypertensive target organ damage. J Hypertens 2004;22:1731-7.

35 Strain WD, Chaturvedi N, Leggetter S, et al. Ethnic differences in skin microvascular function and their relation to cardiac target-organ damage. $J$ Hypertens 2005;23:133-40.

36 Van Popele NE, Grobbee DE, Bots ML, et al. Association between arterial stiffness and atherosclerosis. The Rotterdam study. Stroke 2001;32:454-60.

37 Gladdish S, Rajkumar C. Prevention of cardiac disease in the elderly. J Cardiovasc Risk $2001: 8: 271-7$.

38 McEniery YCM, Wallace S, Mackenzie IS, et al. C-reactive protein is associated with arterial stiffness in apparently healthy individuals. Arterioscle Thromb Vasc Biol 2004;24:969-74.

39 Oren A, Vos LE, Uiterwaal CS, et al. Aortic stiffness and carotid intimal-media thickness: two independent markers of subclinical vascular damage in young adults? Eur J Clin Invest 2003;33:949-54.

40 Girerd X, Giannattasio C, Moulin C, et al. Regression of radial artery wall hypertrophy and improvement of carotid artery compliance after long-term antihypertensive treatment in elderly patients. J Am Coll Cardiol 1998;31:1064-73

41 Benetos A, Cambien F, Gautier S, et al. Influence of the angiotensin 2 type 1 receptor gene polymorphism on the effects of perindopril and nitrendipine on arterial stiffness in hypertensive individuals. Hypertension 1996;25:1034-41.

42 Chen CH, Ting CT, Lin SJ, et al. Different effects of fosinopril and atenolol on wave reflections in hypertensive patients. Hypertension 1996;28:1081-4.

43 VanBortel LM, Spek JJ, Balkestein EJ, et al. Is it possible to develop drugs that act more selectively on large arteries? J Hypertens 1999;17:701-5.

44 Ferrier KE, Muhlmann MH, Bagnet JP, et al. Intensive cholesterol reduction lowers blood pressure and improves large artery stiffness in ISH. J Am Coll Cardiol 2002:9:1020-5.

45 Dart AM, Gatzka CD, Cameron JD, et al. Large artery stiffness is not related to plasma cholesterol in a trial of subjects with hypertension. Arterioscler Thromb Vas Biol 2002;24:962-8

46 Harris TB, Ferrucci L, Tracy RP, et al. Associations of elevated interleukin-6 and C-reactive protein levels with mortality in the elderly. Am J Med 1999;106:506-12

47 Cameron JD, Rajkumar C, Kingwell BA, et al. Higher systemic arterial compliance is associated with greater exercise time and lower blood pressure in a young older population. J Am Geriatr Soc 1999;47:653-6.

48 Miyachi M, Kawano H, Sugawara J, et al. Unfavourable effects of resistance training on central arterial compliance: a randomized intervention study. Circulation 2004; 110:2858-63.

49 Ferrier KE, Waddell TK, Gatzka CD, et al. Aerobic exercise training does not modify large-artery compliance in isolated systolic hypertension. Hypertension $2001 ; 38: 222-6$

50 Wargas $\mathbf{R}$, Wroblewoka B, Rego A, et al. Oestradiol inhibits smooth muscle cell proliferation in pig coronary arteries. Br J Pharmacol 1993;109:612-17.

51 McGrath BP, Liang YL, Teede HJ. Age related deterioration in arterial structure and function in post menopausal women. Arterioscler Thromb Vasc Biol 1998; 18:1149-56.

52 Rajkumar C, Kingwell BA, Cameron J, et al. Hormonal therapy increases arterial compliance in postmenopausal women. J Am Coll Cardiol 1997;30:350-6.

53 Waddell TK, Rajkumar C, Cameron JD, et al. Withdrawal of hormonal therapy for 4 weeks decreases arterial compliance in post menopausa women. J Hypertens 1999;17:413-18.

54 Dockery F, Agarwal S, Donaldson M, et al. Androgen suppression in men leads to increased arterial stiffness and hyperinsulinaemia. Clin Sci 2003;104:195-201.

55 Dockery F, Donaldson M, Fernandez S, et al. Relationship between androgens and arterial stiffness indices in men and women. J Am Geriatr Soc 2003:51:1627-32.

56 Nestel PJ, Pomeroy SE, Sasahara T, et al. Arterial compliance in obese subjects is improved with dietary plant $\mathrm{n}-3$ fatty acid from flax seed oil. Arterioscler Thromb Vasc Biol 1997; 17:1163-70.

57 Nestel PJ. Fish oil and cardiovascular disease: lipid and arterial function. Am J Clin Nutr 2000;71:228-31. 
58 Chin JPF, Gust AP, Nestel PJ, et al. Marine fish oils dose dependently inhibit vasoconstriction of forearm resistance vessels in humans. Hypertension 1993;21:22-8.

59 McVeigh GE, Brennan GM, Cohn JN, et al. Fish oils improves arterial compliance in type 2 diabetics. Arterioscler Thrombo Vasc Biol 1994; 14:1425-9.

60 Vander Schovw YT, Piipe A, Lebrun CE, et al. Higher usual dietary intake of phytoestrogens is associated with lower aortic stiffness in postmenopausal females. Arterioscle Thromb Vasc Biol 2002;22:1316-22.

61 Teede HJ, McGrath BP, Desilva L, et al. Isoflavins reduce arterial stiffness: a placebo controlled study in males and post menopausal females. Arterioscler Thromb Vasc Biol 2003;23:1066-73.

62 Zhan X, Shu X O, Gao Y-T, et al. Soy food consumption is associated with lower risk of coronary heart disease in Chinese women. $J$ Nutr 2003; 133:2874-8.
63 Gates PE, Tanana H, Hiatt WR, et al. Dietary sodium restriction rapidly improves large elastic artery compliance in older adults with systolic hypertension. Hypertension 2004;44:35-41.

64 Eskurza I, Monahan KD, Robinson JA, et al. Ascorbic acid does not affect large elastic artery compliance, central blood pressure in young and older men. Am J Physiol Heart Circ Physiol 2004;286:1528-34.

65 McVeigh GE, Morgan DJ, Finkelstein SM, et al. Vascular abnormalities associated with long-term cigarette smoking identified by arterial waveform analysis. Am J Med 1997;102:227-31.

66 Mahmud A, Feely J. Arterial stiffness and the renin-angiotensin-aldosterone system. J Renin Angiotensin Aldosterone Syst 2004;5:102-8.

67 Kass DA, Shapiro EP, Kawaguchi M, et al. Improved arterial compliance by a novel advanced glycation end-product crosslink breaker. Circulation 2001; 104:1464-70.

68 Grundy SM. Age as a risk factor: you are as old as your arteries. Am J Cardiol 1999;83:1455-7.

$\mathrm{T}$ his month's web trawl examines two UK based web sites, which detail the work of organisations aiming to improve the quality of care delivered to patients within the National Health Service.

http://www.rcplondon.ac.uk/college/ceeu/ This, in fact, in not a web site in its own right, but one of a series of pages from the Royal College of Physicians web site, from their Clinical Effectiveness and Evaluation Unit (CEEU) section. The CEEU aims to improve the quality of care delivered to patients within the NHS, and its web pages, accessed from within their own home page on the RCP web site, give details of its activities. An outline of the work of the CEEU is provided, followed by a series of links to pages detailing the work currently being carried out, and also details of completed projects. Projects undertaken cover a wide variety of disorders, and many are undertaken in collaboration with other groups. For completed projects, links to detailed reports are provided, along with lists of papers that have been published based on the work undertaken. As each project's individual set of pages appear to be updated separately, it is difficult to comment on whether they are all up to date, but in general most appear to have been updated within the past 12 months. The many health professionals in the UK who are already involved in projects run by the CEEU will almost certainly be aware of these pages, but they are likely to be of interest also to some working in related fields, and also potentially to medical and other students wishing to gain an understanding of how research is performed to improve quality of care.

http://www.ncchta.org This is the web site of the NHS Health Technology Assessment (HTA) Programme. The programme provides detailed information on the cost and effectiveness of tests and treatments to those concerned with making decisions regarding their use in the NHS. From the home page, the user may navigate to a series of pages detailing current and previous work undertaken. For those projects now completed, reports may be freely downloaded in pdf format, although the option is also available to purchase a paper copy. The work undertaken covers the whole range of medical and surgical specialties, and the reports available will therefore be of interest to healthcare professionals working in most, if not all disciplines. A further link takes the user to a page entitled funding opportunities, from where application can be made to participate in various named projects and forms may also be downloaded to apply for funding to undertake clinical trials. Although largely aimed at those working within the NHS, the web site also provides pages specifically for members of the public, where details are given of ways they may become involved with the work of the HTA programme. The information on each page is updated regularly and every page appears to be current. Overall, the web site provides a wealth of healthcare information, and will be of interest to both health professionals and members of the public alike.

Robyn Webber Web Editor 